\section{Screening for PTSD}

We read with great interest the article by Brewin et al (2002). The authors examined the efficiency of the 10-item version of the Trauma Screening Questionnaire (TSQ) in detecting post-traumatic stress disorder (PTSD). In our opinion, the scale design has some limitations which may have a negative influence on its practical application.

First, the TSQ contains five reexperiencing items and five arousal items, but not the avoidance and numbing symptoms. According to DSM-IV diagnostic criteria for PTSD (American Psychiatric Association, 1994), the patient requires the presence of at least one reexperiencing symptom (criterion B), three avoidance symptoms (criterion C), and two arousal symptoms (criterion D). The criterion $\mathrm{C}$ is the least frequently met criterion but critically significant to the diagnosis of PTSD (Maes et al, 1998). Some trauma survivors, who express most PTSD symptoms, do not fulfil the avoidance criterion and are diagnosed as having 'partial' PTSD. Other briefer screening instruments, such as the four-item SPAN (Meltzer-Brody et al, 1999) or the seven-item scale by Breslau et al (1999), place much weight on the avoidance and numbing symptoms. Therefore, this specific item composition may influence the efficiency of the TSQ.

Second, the TSQ uses the frequency threshold allied to a 'yes/no' response format. Although comparison of scores derived by frequency and by severity indicated a degree of similarity, the severity dimension might provide better discrimination than the frequency dimension (Meltzer-Brody et al, 1999). In our clinical experience, subjects can score the severity variable more accurately than the frequency ones (Chen et al, 2001). The item selection and scoring method have greater influence on the efficacy of the rating scale.

American Psychiatric Association (1994) Diagnostic and Statistical Manual of Mental Disorders (4th edn) (DSM-IV). Washington, DC: APA.

Breslau, N., Peterson, E. L., Kessler, R. C., et al (1999) Short screening scale for DSM-IV posttraumatic stress disorder. American Journal of Psychiatry, I56,

908-911.

Brewin, C. R., Rose, S., Andrews, B., et al (2002) Brief screening instrument for post-traumatic stress disorder. British Journal of Psychiatry, 18I, 158-162.

Chen, C. H., Lin, S. K., Tang, H. S., et al (200I) The Chinese version of the Davidson Trauma Scale: a practice test for validation. Psychiatry and Clinical Neuroscience, 55, 493-499.
Maes, M., Delmeire, L., Schotte, C., et al (1998) Epidemiologic and phenomenological aspects of post-traumatic stress disorder: DSM-III-R diagnosis and diagnostic criteria not validated. Psychiatry Research, 81, 179-193.

Meltzer-Brody, S., Churchill, E. \& Davidson, J. R.T. (1999) Derivation of the SPAN, a brief diagnostic screening test for post-traumatic stress disorder. Psychiatry Research, 88, 63-70.

M.-L. Lu, W. W. Shen Department of Psychiatry, Taipei Medical University - Wan Fang Hospital, No. III, Hsin-Long Road, Sec. 3, Taipei, Taiwan

Author's reply: Drs Lu and Shen claim that our Trauma Screening Questionnaire (TSQ; Brewin et al, 2002) is flawed because it omits avoidance and numbing symptoms and asks about symptom frequency using a simple 'yes/no' response format. It is puzzling then that the performance of the TSQ is superior to that of all comparable screening measures, including ones that follow Lu and Shen's recommendations. Their views are clearly contradicted by the data from the two studies we reported. Our reasons for designing the TSQ in the way we did were based on empirical and practical rather than theoretical considerations. In our original article we discussed some general principles for designing successful screening instruments, whereas $\mathrm{Lu}$ and Shen's comments seem more relevant to a diagnostic instrument. The two types of measure tend to be administered by different professionals, under different circumstances, and with different aims in mind. It seems to us that, as a screening instrument, what the TSQ gains in simplicity and clarity more than compensates for the absence of symptoms that may be difficult to understand and judgements that may be difficult to make.

Brewin, C. R., Rose, S., Andrews, S., et al (2002) Brief screening instrument for post-traumatic stress disorder. British Journal of Psychiatry, 18I, 158-162.

C. R. Brewin Sub-Department of Clinical Health Psychology, University College London, Gower Street, London WCIE 6BT, UK

\section{Transcultural psychiatry}

Drs Bhui \& Bhugra (2002) address the interesting area of explanatory models for mental distress. They do not, however, justify why we should elicit patients' explanatory models. The notion that members of a specific cultural group hold similar ideas about illness and that culture can be distilled into a set of specific 'beliefs' is considered outdated and oversimplified by medical anthropologists. Kleinman (1980) points out that explanatory models are idiosyncratic and are justifications for actions rather than causes. Bhui \& Bhugra themselves cite Williams \& Healy (2001), who point out that it is difficult to distil a single set of causal explanations that might relate to behaviour, diagnosis or adherence to medication treatment.

The assertion by Bhui \& Bhugra that shared understanding of illness between patient and healer distinguishes traditional healing systems from Western biomedicine is simply not borne out by the anthropological literature. In many systems of traditional healing, patients have little understanding of how the treatment 'works' and it is the healer who holds highly esoteric knowledge. There is little empirical evidence that eliciting explanatory models improves satisfaction. The one study cited (Callan \& Littlewood, 1998 ) in fact found that $79 \%$ of patients with divergent explanatory models (a comparison of the explanatory models of doctors and patients) were satisfied with psychiatric services.

Of course, patients do have cultural understanding of their illness but this may not be very sophisticated and may not directly relate to decisions about treatments. There is a large amount of data from medical anthropological research which suggests that treatment choice is determined primarily by social and political factors rather than by underlying explanatory models (Pelto \& Pelto, 1997). Even a study using the Explanatory Model Interview Catalogue (Weiss et al, 1992) among leprosy patients suggests that those who held theories of humoral imbalance rather than biomedical theories of infection, sanitation and hygiene had the best biomedical clinic attendance records for leprosy treatment. In terms of treatment outcomes patients may not be interested in how a treatment works (Last, 1981) as long as it does work. The weight of empirical evidence suggests that people are keen to utilise biomedical treatments regardless of their cultural beliefs without giving up traditional explanations of illness. In fact, as my own data (Dein, 2001) suggest, among Asian psychiatric patients, biomedical and traditional models of illness are held concurrently and informants agree that biomedical 